Transferring the film from the liquid substrate to a solid substrate has proven difficult. The researchers tried two techniques. For the investigation of the upper side of the film, a microscope slide can be slid into the liquid just under the monocrystalline film to lift it slowly. With this method, the film broke, but crystal fragments of more than $2 \mathrm{~mm}^{2}$ area were preserved. To investigate the underside of a crystal, tape affixed to a sample holder was drawn down until it gently touched the monocrystalline film. Then the film was lifted from the liquid. With this method, 7-mm-diameter crystals could be separated without breaking.

The researchers concluded that this is a potentially useful approach for producing large photonic single crystals, which are the prerequisite for the preparation of resonators or, more generally, for wave guiding in photonic structures.

ANDREI A. ELISEEV

\section{Predetermined Chiroptical \\ Properties Expressed on Rosette Nanotubes}

Chirotechnology is an emerging field with the potential for application in the design of sensors, chiral cholesteric phases, catalysts, and materials with electromagnetic, optoelectronic, information-storage, display-system, nonlinear optical, and novel chiral light-emitting applications. Induction of nonrandom symmetry breaking in supramolecular systems by external means, such as chiral vortex forces, electron transfer, and photoswitches, have the advantages of predictability and reproducibility. As a step toward a better understanding of nature's approach to generating supramolecular structures with predefined stereochemistry and architecture, researchers at the H.C. Brown Chemistry Laboratory, Purdue University, have extended the functionality of self-assembled helical rosette nanotubes by imparting on them adjustable chiroptical properties.

As reported in the August 24 issue of the Journal of the American Chemical Society, a team of Purdue University researchers led by $\mathrm{H}$. Fenniri induced supramolecular chirality in rosette nanotubes (see figure) using two methods: by inducting symmetry breaking in a preexisting racemic mixture of $\mathrm{M}$ - and P-helical rosette nanotubes; and by employing a chiral promoter on a prochiral molecular module to trigger the hierarchical self-assembly of chiroptical rosette nanotubes. Both methods employ promoters that transfer their molecular chirality to the supramolecular ensemble as well as stabilize the nanotube assembly.

Rosette nanotubes are a class of organic nanostructures whose aqueous selfassembly is entropically driven. The single building block in these assemblies is a bicyclic molecule-compound 1-with a hydrogen-bond donor-donor-acceptor array on one side, an acceptor-acceptor-donor array on the other, and a crown ether substituent (see figure). Rosettes, which consist of a six-membered macrocycle held together by 18 hydrogen bonds, stack to form a nantobube with a central pore and peripheral channels formed by the crown ethers. As expected by Fenniri and co-workers, induced circular dichroism (ICD) resulted from the binding of a chiral amino acid (either L- or D-alanine) to the prochiral crown ether. Transmission electron microscope (TEM) studies of concentrated rosette nanotubes in the absence of any amino-acid promoter confirmed a tube diameter of about $4 \mathrm{~nm}$. Dynamic light scattering (DLS) showed that the average hydrodynamic radius of the nanotubes is $32.5 \mathrm{~nm}$, which indicates that the nanotubes are composed of about 140 rosettes, on average, if $4.5 \AA$ between stacks is assumed. DLS and TEM were used to confirm nanotube formation in the presence of L-alanine. The researchers believe that their investigations demonstrate not only self-assembly in the presence of L-alanine but that the assembly is expressing collectively the promoter's molecular chirality at the nanotube level.

The research team classified 20 promoters as inducing a strong, a medium, or no circular dichroism (CD) signal. All active promoters with the same chirality were found to induce the same helicity. For L-alanine analogues, primary ammonium or carboxylate functions were deemed essential for supramolecular chirality induction. The inductive effect was and small-angle $x$-ray scattering (SAXS)

shown to be both promoter-specific and highly sensitive to minor structural variations. The researchers believe that the inability of a promoter to induce chirality is due to nanotube destabilization, weak interactions, or an achiral structure. Nanotubes were found to disassemble at low concentration $[\mathbf{1}(0.04 \mathrm{mM})]$ in the absence of any promoter. Monitoring of the $\mathrm{CD}$ as $\mathbf{1}(0.04 \mathrm{mM})$ was titrated with L-alanine showed that the transition from racemic to helical rosette nanotubes occurs in the range of 4-30 equivalents of L-alanine. Therefore, almost all of the binding sites must be promoter-occupied for a complete transition-an all-or-none response that the researchers said is at variance with a classical "sergeant-and-soldiers" mechanism whereby a few chiral species determine the overall chirality.

The researchers demonstrated several promoter phenomena: enantiospecificity, in which all of the $\mathrm{L}$ amino acids were found to induce the exact opposite CD response as their D-isomers; reversibility, in which heating caused the ICD to decrease sharply but cooling restored it to $70 \%$ within minutes and to $100 \%$ within $24 \mathrm{~h}$; and dominant/recessive behavior, in which the addition of a large excess of D-alanine to $\mathbf{1}$ preequilibrated with L-alanine resulted in an ICD identical to 1 preequilibrated with D-alanine. Analysis of the $P \leftrightarrow M$ equilibrium suggests to the researchers that the promoters "within their sterically matching nanotubes must interact cooperatively not only to stabilize but also to feed their nanotube host with additional rosette stacks and 1-promoter complexes." The researchers propose these interactions are electrostatic bonds between the carboxylate and ammonium groups.

The researchers found two pathways

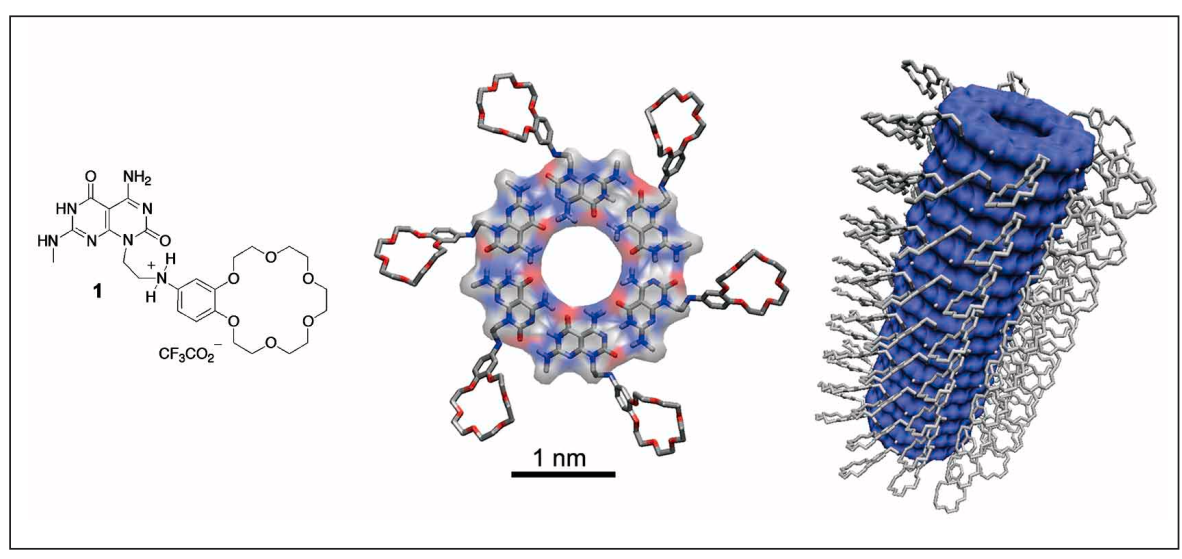

Figure. Compound 1 (left) self-assembles to form a rosette assembly (middle), which then self-organizes into a rosette nanotube (right). 
that lead to the formation of chiroptical rosette nanotubes. At millimolar concentrations and in the absence of a promoter, 1 self-assembles into a racemic mixture of helical rosette nanotubes. An instantaneous transition from racemic to homochiral helical nanotubes accompanies the addition of a promoter. At micromolar concentrations, $\mathbf{1}$ is unassembled but addition of a promoter triggers selfassembly into rosette nanotubes with a helicity predefined by the promoter. The supramolecular reactions involved must first involve molecular recognition between 1 and the promoter.

Fenniri and co-workers believe that when viewed as a model process by which predefined molecular properties are expressed at the macromolecular level through programmable stereospecific self-assembly.

STEVEN TROHALAKI

\section{Acoustical Memory Observed in $\mathrm{LiNbO}_{3}$}

Michael McPherson and colleagues from the National Center for Physical Acoustics at the University of Mississippi have observed acoustical memory in $\mathrm{LiNbO}_{3}$ crystals. An acoustical tone burst was stored inside the crystal and reemitted at a later time. They report in the September issue of Physical Review Letters that the strength of the memory signal is directly related to the dielectrical properties of the ferroelectric material, and is sensitive to both temperature and frequency. Acoustical memory may be useful for investigating energy exchange processes in ferroelectrics, they said.

Cylinders of bulk $\mathrm{LiNbO}_{3}$ ranging from $2.4 \mathrm{~cm}$ to $3.9 \mathrm{~cm}$ in length and from $2.5 \mathrm{~cm}$ to $4.5 \mathrm{~cm}$ in diameter were used to demonstrate the effect, where the acoustical longitudinal mode propagation along the [001] axis corresponds with the piezoelectrically active direction. A $39^{\circ}$ rotated Y-cut 30-MHz transducer was mounted to the top of the sample and a capacitive receiver was mounted to the bottom to capture the resulting signal. A synthesizer function generator produced sinusoidal tone bursts approximately $5 \mu$ s in duration. To verify the results, the experiment was repeated in which the configuration involved the removal of the capacitive receiver, causing the transducer to act as both emitter and receiver. In another configuration, a secondary transducer was mounted to the opposite face of the sample, as a standard two-transducer setup. The samples were tested at various temperatures ranging from $24^{\circ} \mathrm{C}$ to $46^{\circ} \mathrm{C}$ and within a signal frequency range of 16-26 $\mathrm{MHz}$. The researchers found that the acoustical memory effect was sensitive to both temperature and frequency, and closely related to the dielectrical properties of the sample, possibly due to the pinning and relaxation of domain walls within the crystal. Within the frequency range, extrema appear in the acoustical memory amplitude, implying a memory center size. The researchers estimate these memory centers to be 140-230 $\mu \mathrm{m}$ for the observed peaks, which corresponds to the size of ferroelectric domains in lithium niobate.

CHRISTINE RUSSELL

\section{Removal Rates in Chemical- Mechanical Planarization of Copper and Silica Surfaces Increase with Particle Concentration When Using Dilute Solutions}

Chemical-mechanical planarization (CMP), a polishing process commonly used in the fabrication of wafers and other components in the electronics industry, has removal rates usually proportional to the applied pressure and velocity of the surfaces in contact. This proportionality is defined by the Preston coefficient, and may be linear or follow a power law. The Preston coefficient varies depending on reaction kinetics, solution concentration, or surface properties. A group of scientists from the Motorola Digital DNA Laboratories and the AMDMotorola Technology Alliance in Austin, Texas, studied the effect of particle concentration on the removal rates, and reported their results in the October issue of Electrochemical and Solid-State Letters. $\mathrm{K}$. Cooper and colleagues studied dilute solutions where particle size is negligible compared to the mean particle separation distance, expecting the Preston coefficient to be proportional to the frequency of collisions. In this regime taking into account volumetric considerations, the Preston coefficient has a term proportional to the cubic root of the weight of solids ( $\mathrm{wt}^{\mathrm{O}} \mathrm{0}^{1 / 3}$ ) present in the solution. In order to prove this dependence, the scientists conducted experiments on silica and copper films. Silicon dioxide films $450 \mathrm{~nm}$ thick were deposited by chemical vapor deposition (CVD) on 200-mm silicon substrates. Subsequently, the wafers were polished on a rotational tool for $60 \mathrm{~s}$ at constant pressure and velocity using a solution of silica particles with a mean size of $120 \mathrm{~nm}$. Measurements of the film thickness before and after polishing were obtained with a spectrophotometer. Copper films $1.2 \mu \mathrm{m}$ thick were deposited by physical vapor deposition (PVD) on a $100 \mathrm{~nm} \mathrm{Cu}$ seed, then annealed for complete recrystallization and polished using a solution of sili- ca particles with a mean size of $40 \mathrm{~nm}$. Thicknesses were measured with a calibrated four-point probe.

Data showed that in both cases the removal rates follow linear behavior with respect to pressure and velocity. To determine the effect of abrasive solutions on the oxidation and removal rates on copper, measurements were taken with a voltammeter in a Pt disk electroplated with $10 \mu \mathrm{m}$ of $\mathrm{Cu}$. The disk was rotated at $300 \mathrm{rpm}$ and polished with a pressure of $50 \mathrm{kPa}$ using solutions with $<1 \mathrm{wt} \%$ and without abrasive particles. During polishing without abrasive particles, a passive oxide layer appeared. On the other hand, particles provided an increase in current density and anodic slope, suggesting that the mechanism of oxidation followed by mechanical removal was apparent. The removal rates for both silica and copper wafers proved to be proportional to $w \mathrm{t}^{\mathrm{O}} \mathrm{o}^{1 / 3}$ with both materials having different critical onset concentrations, and with silica having an onset concentration about six times greater than that for copper. The researchers attribute this to differences in kinetics and removal mechanisms. Copper oxides have poorer adhesion to copper substrates than silica has to silicon, making them easier to remove. Planarity of wafers is improved by increasing slurry concentration, with surface roughness decreasing down to 3.5 times that of the unpolished surface.

SIARI S. SOSA

\section{Method Developed for Asymmetric Patterning of Nanospheres}

One approach to fabricating functional nanoscale architectures is the selfassembly of "high-information-content" molecules and nanoparticles modified with a homogeneously distributed layer of molecules. However, the spatial homogeneity at the nanoparticle surface limits both the structural diversity and functional complexity of nanoscale architectures that can be contemplated. It is in this context that researchers at University College Dublin, Ireland have developed a strategy for patterning nanoparticles in order to realize the full potential of this approach. L. Nagle and colleagues have demonstrated the patterning of nanospheres by assembling gold nanoparticles, $16 \mathrm{~nm}$ in diameter, on only one side of a larger silica or latex nanosphere, $200 \mathrm{~nm}$ and $500 \mathrm{~nm}$ in diameter, respectively. Their strategy for patterning nanospheres uses ultrafiltration technology.

As reported in Nano Letters (Web release October 10, 2002), the key to modifying one half of a nanosphere lies 